\title{
The Perioperative Anticoagulant Use for Surgery Evaluation (PAUSE) Study for Patients on a Direct Oral Anticoagulant Who Need an Elective Surgery or Procedure: Design and Rationale
}

James D. Douketis ${ }^{1}$ Alex C. Spyropoulos ${ }^{2} \quad$ Julia M. Anderson ${ }^{3}$ Donald M. Arnold ${ }^{1}$ Shannon M. Bates ${ }^{1}$ Mark Blostein ${ }^{4}$ Marc Carrier $^{5}$ Joseph A. Caprini ${ }^{6}$ Nathan P. Clark ${ }^{7}$ Michiel Coppens ${ }^{8}$ Francesco Dentali $^{9}$ Joanne Duncan ${ }^{1}$ Peter L. Gross ${ }^{1}$ Jeannine Kassis ${ }^{10}$ Stephen Kowalski ${ }^{11}$ Agnes Y. Lee ${ }^{12}$ Gregoire Le Gal ${ }^{13}$ Geneviève Le Templier ${ }^{14} \mathrm{Na} \mathrm{Li}^{1} \quad$ Elizabeth MacKay $^{15}$ Vinay Shah ${ }^{16}$ Sudeep Shivakumar ${ }^{17}$ Susan Solymoss ${ }^{4}$ Frederick A. Spencer ${ }^{1}$ Summer Syed ${ }^{18}$ Alfonso J. Tafur ${ }^{6}$ Thomas Vanassche ${ }^{19}$ Thomas Thiele ${ }^{20}$ Cynthia Wu ${ }^{21}$ Erik Yeo ${ }^{22}$ Sam Schulman ${ }^{1}$

1 Department of Medicine, McMaster University, Hamilton, Canada

2 Department of Medicine, Hofstra Northwell School of Medicine, Northwell Health at Lenox Hill Hospital, New York,

New York, United States

${ }^{3}$ Department of Medicine, University of Edinburgh, Edinburgh, United Kingdom

${ }^{4}$ Department of Medicine, McGill University, Montreal, Canada

5 Ottawa Hospital Research Institute, University of Ottawa,

Ottawa, Canada

${ }^{6}$ NorthShore University Health Systems, Evanston, Illinois, United States

${ }^{7}$ Department of Pharmacy, Kaiser Permanente Colorado, Aurora, Colorado, United States

${ }^{8}$ Department of Vascular Medicine, Academic Medical Center, University of Amsterdam, Amsterdam, The Netherlands

${ }^{9}$ Department of Medicine and Surgery, Insubria University, Varese, Italy

10 Department of Medicine, Université de Montréal, Montreal, Canada

${ }^{11}$ Department of Anesthesiology, University of Manitoba, Winnipeg, Canada

12 Department of Medicine, University of British Columbia, Vancouver, Canada

\footnotetext{
13 L'Institut du Savoir Montfort, L'Hopital Monfort, Ottawa, Ontario, Canada

${ }^{14}$ Department of Internal Medicine, Centre Hospitalier Universitaire de Sherbrooke, Quebec, Canada

${ }^{15}$ Department of Medicine, University of Calgary, Calgary, Canada

${ }^{16}$ Department of Medicine, Henry Ford Hospital, Detroit, Michigan, United States

17 Department of Medicine, Dalhousie University, Halifax, Canada

${ }^{18}$ Department of Anesthesiology, Hamilton Health Sciences, McMaster University, Hamilton, Canada

${ }^{19}$ Department of Cardiovascular Sciences, Universitaire Ziekenhuizen Leuven, Leuven, Belgium

20 Institute for Immunology and Transfusion Medicine, Universitätsmedizin Greifswald, Greifswald, Germany

${ }^{21}$ Department of Medicine, University of Alberta, Edmonton, Canada

22 Department of Medicine, University of Toronto, Toronto, Canada

Address for correspondence James D. Douketis, MD, St. Joseph's Healthcare Hamilton, Room F-544, 50 Charlton Avenue East, Hamilton, Ontario L8N 4A6, Canada (e-mail: jdouket@mcmaster.ca).
}

\section{Abstract}

received

August 11, 2017

accepted after revision

September 12, 2017
Background The perioperative management of patients who take a direct oral anticoagulant (DOAC) for atrial fibrillation and require treatment interruption for an elective surgery/procedure is a common clinical scenario for which best practices are uncertain. The Perioperative Anticoagulant Use for Surgery Evaluation (PAUSE) study is designed to address this unmet clinical need. We discuss the rationale for the PAUSE design and analysis plan as well as the rationale supporting the perioperative DOAC protocol.

Methods PAUSE is a prospective study with three parallel cohorts, one for each DOAC, to assess a standardized but patient-specific perioperative management protocol for

Septenber 12,2017 
Keywords

- surgery

- thrombosis

- clinical trials

- oral anticoagulants
DOAC-treated patients with atrial fibrillation. The perioperative protocol accounts for DOAC type, patient's renal function and surgery/procedure-related bleeding risk. The primary study aim is to demonstrate the safety of the PAUSE protocol for the perioperative management of each DOAC. The secondary aim is to determine the effect of the pre-procedure interruption on residual anticoagulation when measured by the dilute thrombin time for dabigatran and anti-factor Xa levels for rivaroxaban and apixaban. The study hypothesis is that the perioperative management protocol for each DOAC is safe for patient care, defined by expected risks for major bleeding of $1 \%$ ( $80 \%$ power to exclude $2 \%$ ), and for arterial thromboembolism of $0.5 \%(80 \%$ power to exclude $1.5 \%$ ) in each DOAC group.

Conclusion The PAUSE study has the potential to establish a standard-of-care approach for the perioperative management of DOAC-treated patients. The PAUSE management protocol is designed to be easily applied in clinical practice, as it is standardized and also patient specific.

\section{Introduction}

The management of patients who are receiving a direct oral anticoagulant (DOAC) and require an elective surgery or invasive procedure is an increasingly common and challenging clinical scenario. ${ }^{1}$ The clinical scope of this problem is increasing, as DOACs are replacing warfarin as the recommended first-line anticoagulant treatment option for stroke prevention in patients with atrial fibrillation. ${ }^{2,3}$ Moreover, the number of patients with atrial fibrillation is increasing due to an aging population, and such older patients are more likely to require a surgery/procedure than younger patients. ${ }^{4}$ It is estimated that 12 to $15 \%$ of patients with atrial fibrillation will require anticoagulant interruption for an elective surgery/ procedure annually. ${ }^{5,6}$ The perioperative management of such patients is pertinent to many clinicians, including internists, surgeons, anaesthetists, family physicians, and dentists., ${ }^{4,7}$

Managing such patients is challenging because evidencebased guidelines to inform best practices are lacking. Although the American and European Societies of Regional Anesthesia, the American College of Cardiology and the American Heart Association have provided guidelines for the perioperative management of DOAC-treated patients, these documents can only be considered expert-opinion guidance, as they are not anchored on findings from prospective studies that assess perioperative management strategies. ${ }^{8-10}$ Only one prospective study, to our knowledge, has assessed a standardized perioperative management approach in patients who required an elective surgery/procedure, but this study was limited as it included only patients on dabigatran. ${ }^{11}$

Given the magnitude of the clinical problem coupled with the paucity of practice-informing prospective clinical studies, the Perioperative Anticoagulant Use for Surgery Evaluation (PAUSE) study (NCT02228798) was designed. The primary study aim is to establish a safe, standardized protocol for the perioperative management of patients with atrial fibrillation who are receiving a DOAC (dabigatran, rivaroxaban or apixaban) and require treatment interruption for an elective surgery/procedure. Herein, we describe the rationale for the PAUSE study design and analysis plan, and elaborate on the reasoning behind the standardized preprocedure DOAC interruption and post-procedure DOAC resumption protocols.

\section{Study Methods}

\section{Study Aims, Design and Rationale}

The primary aim of PAUSE is to demonstrate the safety of a standardized but patient-specific protocol for the perioperative management of DOACs. This protocol is adjusted according to DOAC type, patient's renal function and surgery/ procedure-related bleeding risk to optimize patient safety, and does not involve heparin bridging anticoagulation. ${ }^{1}$ The PAUSE study requires approval by institutional review boards of all participating clinical sites and written informed consent from all participating patients.

We hypothesize that the perioperative management protocol is 'safe for patient care' in patients with atrial fibrillation, defined by an expected low risk for major bleeding (i.e. $1 \%$; $80 \%$ power to exclude $2 \%$ ) and an expected low risk for arterial thromboembolism (i.e. $0.5 \%$; $80 \%$ power to exclude $1.5 \%$ ). These expected risks were based on the available literature at the time of the protocol development (in 2013), which showed perioperative risks of major bleeding of 1 to $3 \%$ and risks of arterial thromboembolism of 0.4 to $1.5 \%$ in warfarinand DOAC-treated patients who, like patients in PAUSE, would have anticoagulant interruption for an elective surgery/procedure and did not receive heparin bridging. ${ }^{12,13}$

The secondary study aim is to determine the effect of the DOAC interruption protocol on the level of residual anticoagulation when measured by DOAC-specific coagulation tests, comprising the dilute thrombin time (dTT) for dabigatran and anti-factor Xa assays for rivaroxaban and apixaban. ${ }^{14}$ For all DOACs, the effect of DOAC interruption on nonspecific coagulation tests, comprising the prothrombin time (PT), the activated partial thromboplastin time (aPTT) and thrombin time (TT), is also assessed. ${ }^{15,16}$ In an exploratory manner, we postulated that the pre-procedure DOAC 
interruption protocol is associated with a high proportion (90\%) of patients with a minimal residual DOAC-associated anticoagulant effect just before a surgery/procedure, which we define as a dTT or anti-factor Xa level $<50 \mathrm{ng} / \mathrm{mL}$.

The PAUSE study uses a prospective cohort design, with three parallel groups for dabigatran, rivaroxaban and apixaban (edoxaban and betrixaban were not available for clinical use when PAUSE was designed), to assess a standardized but patient-specific perioperative management protocol. A nonrandomized, prospective cohort design was chosen based on the following considerations: First, a cohort design is appropriate to assess the safety of a clinical management strategy that is expected to be associated with low risks of adverse clinical events, and when there is sufficient statistical power to exclude clinically important higher risk of events. ${ }^{17,18}$ Although cohort studies have the potential for patient selection bias, for example if presumed high-risk patients are more likely to be excluded, such bias was deemed unlikely in PAUSE because in a precursor perioperative dabigatran study, $90 \%$ of eligible patients consented to participate. ${ }^{11}$ Moreover, the lack of a standard-of-care approach for the perioperative management of DOAC-treated patients would make it unlikely that patient groups would be systematically excluded, especially when the aim of PAUSE is to establish best practices. Second, a randomized trial design was not considered because a true control group would be lacking, as there is no established or de facto standard of care for perioperative DOAC management. For example, a comparator group wherein DOACs are interrupted 5 days pre-procedure in all patients, as is done with warfarin, would be a second experimental arm. Third, a cluster randomized trial design, whereby clinical sites would allocate patients to the PAUSE protocol or usual care, was not considered because usual care would frequently be the same or similar to the PAUSE protocol in some clinical sites, thereby precluding a distinction between the two perioperative management strategies.

\section{Patient Population and Rationale}

All of the following inclusion criteria have to be satisfied for patient study eligibility: (1) age $\geq 18$ years; (2) receiving dabigatran (150 or $110 \mathrm{mg}$ twice daily), rivaroxaban (20 or $15 \mathrm{mg}$ daily) or apixaban (5 or $2.5 \mathrm{mg}$ twice daily) for stroke prevention in atrial fibrillation; (3) undergoing an elective surgery or procedure that requires temporary interruption of anticoagulant therapy and (4) ability to adhere to the preprocedure DOAC interruption protocol. Patients are excluded if they have $\geq 1$ of the following criteria: (1) creatinine clearance $(\mathrm{CrCl})<30 \mathrm{~mL} / \mathrm{min}$ for dabigatran- and rivaroxaban-treated patients, or $\mathrm{CrCl}<25 \mathrm{~mL} / \mathrm{min}$ for apixaban-treated patients, as estimated by the Cockcroft-Gault formula, ${ }^{19}$ as these DOACs are contraindicated if the $\mathrm{CrCl}$ is below these cut-points; (2) cognitive impairment or psychiatric illness that might preclude reliable post-procedure follow-up and documentation of outcome events; (3) inability or unwillingness to provide informed consent and (4) previous participation in the PAUSE study. In PAUSE, we use the Cockcroft-Gault formula to estimate patient eligibility according to renal function because this formula has been used to determine patient eligibility for the randomized trials that assessed DOACs. ${ }^{20-23}$ In everyday practice, it is also recommended to use the Cockcroft-Gault formula to estimate renal function and, in turn, assess patient eligibility for DOACs, and to determine the dose regimen for rivaroxaban. ${ }^{24}$

The PAUSE study focuses on patients with atrial fibrillation because this is the dominant clinical indication for longterm DOAC therapy, and such patients comprise 70 to $80 \%$ of patients who are assessed for perioperative anticoagulant management. ${ }^{25}$ Patients with a mechanical heart valve are excluded because DOACs are contraindicated in such patients, but patients with bioprosthetic heart valves or non-rheumatic valvular heart disease are not excluded as DOACs can be used in such patients. ${ }^{2,3}$ We do not include patients with venous thromboembolism as the sole indication for DOAC therapy because they constitute a separate population who tend to be younger and with fewer comorbidities than patients with atrial fibrillation, and are more likely to develop venous rather than arterial thromboembolic adverse outcomes. ${ }^{26,27}$ Moreover, inclusion of such patients would, in effect, create a dichotomous patient population that would affect the generalizability of the study results.

\section{Classification of Bleeding Risk Associated with Surgery/Procedure and Thromboembolism Risk}

The classification of surgery/procedure types as low bleeding risk or high bleeding risk is an essential part of the preprocedure assessment, as this determines the timing of DOAC interruption and resumption. Procedures classified as high bleeding risk have a longer interruption and resumption interval (2 days) compared with those having a low bleeding risk (1 day). As there is no established classification scheme to distinguish surgery/procedure-associated bleeding risk, the approach used in PAUSE is empiric but based on the bleeding risk classification used in the BRIDGE trial. ${ }^{4}$ In addition, the PAUSE protocol allows flexibility in the procedure-related bleeding risk classification to account for reallife situations. For example, a patient having surveillance colonoscopy would have low-bleeding risk management pre-procedure (1 day off DOAC), but if multiple or large polyps are removed during the procedure, this patient would be allowed to have high-bleeding risk management where DOAC resumption is delayed for 48 to 72 hours. ${ }^{28,29}$

The PAUSE study documents patients' $\mathrm{CHADS}_{2}$ and $\mathrm{CHA}_{2} \mathrm{DS}_{2}$-VASc scores, ${ }^{30,31}$ but these scores do not affect perioperative anticoagulant management. Thus, no patient receives therapeutic-dose heparin bridging in PAUSE, irrespective of the perceived thromboembolic risk. The no-bridging approach adopted in PAUSE is supported by the recent publication of the BRIDGE trial in warfarin-treated patients, ${ }^{4}$ and related studies in DOAC- and warfarin-treated patients, ${ }^{7,32}$ showing that a no-bridging strategy is non-inferior to bridging to prevent thromboembolism and superior to a bridging strategy for the prevention of major bleeding.

Peri-procedural Management Protocol and Rationale The overall aim is to develop a standardized but patientspecific management protocol, which accounts for the DOAC taken, patient's renal function, and surgery/procedure type 
and which is easy to implement in clinical practice. The design of the pre-procedure DOAC interruption protocol, as shown in - Fig. 1, was the most challenging aspect of the protocol development. Particular attention is given to patients having a high bleeding risk surgery/procedure or neuraxial anaesthesia, so that the DOAC interruption interval results in minimal to no residual anticoagulant effect at the time of the surgery/anaesthesia.

\section{Pre-procedure DOAC Interruption Protocol}

Three sources of information were considered in developing the pre-procedure DOAC interruption interval: (1) the elimination half-life of each DOAC, as reported for dose regimens used for stroke prevention in atrial fibrillation; ${ }^{33-36}(2)$ the DOAC manufacturers' recommended pre-procedure interruption intervals and (3) the available literature relating to perioperative management of DOAC-treated patients.

Another issue was whether the pre-procedure DOAC interruption would be expressed as an hour-based (i.e. 24 or $48-$ 72 hours) or day-based interval (1 or 2-3 days) between the last drug dose and the surgery/procedure. We chose the 'daybased interval', comprising 1 day off for a low bleeding risk and 2 days off for a high bleeding risk surgery/procedure because it offered a simple protocol that would be easy to apply in everyday practice and easy to follow by patients. In addition, it allowed a slightly longer DOAC interruption interval than the 'hour-based interval', which would be particularly important to ensure minimal to no residual anticoagulant effect in patients having a high bleeding risk surgery/procedure. ${ }^{37}$

To illustrate the advantage of this approach used in PAUSE, consider a patient with atrial fibrillation $(\mathrm{CrCl}, 60 \mathrm{~mL} / \mathrm{min})$ who is having elective hip replacement on a Monday at $8 \mathrm{Am}$ and is taking dabigatran, $150 \mathrm{mg}$ twice daily, at 9 AM and 6 PM. If the 'hour-based interval' is used, the last dabigatran dose would be Friday 6 PM, to allow a 48-hour interval between the last dose and the surgery. If, on the other hand, the surgery was on a Monday at noon, the last dabigatran dose would be Saturday at 9 Am to have a 48-hour interruption interval. By comparison, the 'day-based interval' would simply mean the last dabigatran dose would be on Friday (2 days off before the surgery) irrespective of the timing of surgery on Monday. If the surgery was on Monday at noon, the PAUSE approach would also allow a longer dabigatran interruption interval of 66 hours (with last dose on Friday 6 PM), instead of a 51-hour interval (with last dose on Saturday $9 \mathrm{Am}$ ). Given the dabigatran elimination half-life of 12 to 14 hours, the 2-day off approach would correspond to approximately five elimination half-lives between the last dose and the time of surgery and would provide reassurance to the surgeon and anesthetist of minimal to no remaining anticoagulant effect.

In patients having a low bleeding risk surgery/procedure, a 1-day off (or 36-42 hours) interval between the last DOAC dose and the surgery/procedure would be acceptable, as there would be a minimal residual anticoagulant at the time of the surgery/procedure effect corresponding to three to four elimination half-lives between the last DOAC dose and the surgery/procedure. In all patients, no DOAC would be taken on the day of the surgery/procedure.

Patient's renal function was also considered in determining the pre-procedure DOAC interruption interval. If a patient was taking dabigatran, which has 75 to $80 \%$ renal clearance, and had a $\mathrm{CrCl} 30$ to $50 \mathrm{~mL} / \mathrm{min}$, the duration of interruption was extended to 2 days in patients having a low bleeding risk procedure and to 4 days in patients having a high bleeding risk procedure. If a patient was taking rivaroxaban or apixaban and had a $\mathrm{CrCl} 30$ to $50 \mathrm{~mL} / \mathrm{min}$, there was no extension in the interruption interval, as these DOACs have only 25 to $33 \%$ renal clearance.

\section{Post-procedure DOAC Resumption Protocol}

As shown in -Fig. 2, DOACs would be resumed 1 day ( $\sim 24$ hours) after a low bleeding risk surgery/procedure and 2 to 3 days ( $\sim 48-72$ hours) after a high bleeding risk surgery/procedure. This flexible, patient-specific approach reflects the variable bleeding risk associated with different surgery/procedure types and is designed to minimize the risk for post-procedure bleeding. Previous studies suggested a high post-procedural bleeding risk if therapeutic-dose heparins are uniformly re-initiated approximately 24 hours after a procedure irrespective of procedural bleeding risk. ${ }^{38}$

For patients in whom DOAC resumption was delayed for 2 to 3 days post-procedure and those who were considered

\begin{tabular}{|c|c|c|c|c|c|c|c|}
\hline \multirow[t]{2}{*}{ DOAC type } & \multirow{2}{*}{$\begin{array}{c}\text { Surgery/procedure } \\
\text { bleed risk }\end{array}$} & \multicolumn{5}{|c|}{ Pre-procedure interruption timing of DOAC } & \multirow[b]{2}{*}{ Day 0} \\
\hline & & Day -5 & Day -4 & Day -3 & Day -2 & Day -1 & \\
\hline \multirow{2}{*}{$\begin{array}{l}\text { Dabigatran } \\
(\mathrm{CrCl} \geq 50 \mathrm{~mL} / \mathrm{min})\end{array}$} & High & & & & & & \multirow{8}{*}{$\begin{array}{c}\text { No DOAC } \\
\text { taken on the } \\
\text { day of } \\
\text { surgery/proce } \\
\text { dure }\end{array}$} \\
\hline & Low & & & & & & \\
\hline \multirow{2}{*}{$\begin{array}{l}\text { Dabigatran } \\
(\mathrm{CrCl}<50 \mathrm{~mL} / \mathrm{min})\end{array}$} & High & & & & & & \\
\hline & Low & & & & & & \\
\hline \multirow[t]{2}{*}{ Rivaroxaban } & High & & & & & & \\
\hline & Low & & & & & & \\
\hline \multirow[t]{2}{*}{ Apixaban } & High & & & & & & \\
\hline & Low & & & & & & \\
\hline
\end{tabular}

Fig. 1 PAUSE protocol for DOAC interruption before a surgery/procedure. Solid arrows indicate usual DOAC dose taken on those days; shaded area indicates no DOAC taken on those days. DOAC, direct oral anticoagulant; PAUSE, the Perioperative Anticoagulant Use for Surgery Evaluation. 


\begin{tabular}{|c|c|c|c|c|c|c|c|}
\hline \multirow[t]{2}{*}{ DOAC type } & \multirow{2}{*}{$\begin{array}{c}\text { Surgery/procedure } \\
\text { bleed risk }\end{array}$} & \multirow[b]{2}{*}{ Day 0} & \multicolumn{5}{|c|}{ Post-procedure resumption timing of DOAC } \\
\hline & & & Day $+1^{\dagger}$ & Day +2 & Day +3 & Day +4 & Day +5 \\
\hline \multirow{2}{*}{$\begin{array}{l}\text { Dabigatran } \\
(\mathrm{CrCl} \geq 50 \mathrm{~mL} / \mathrm{min})\end{array}$} & High & \multirow{8}{*}{$\begin{array}{l}\text { No DOAC taken } \\
\text { on the day of } \\
\text { surgery or } \\
\text { procedure }\end{array}$} & & & & & \\
\hline & Low & & & & & & \\
\hline \multirow{2}{*}{$\begin{array}{l}\text { Dabigatran } \\
(\mathrm{CrCl}<50 \mathrm{~mL} / \mathrm{min})\end{array}$} & High & & & & & & \\
\hline & Low & & & & & & \\
\hline \multirow[t]{2}{*}{ Rivaroxaban } & High & & & & & & \\
\hline & Low & & & & & & \\
\hline \multirow[t]{2}{*}{ Apixaban } & High & & & & & & \\
\hline & Low & & & & & & \\
\hline
\end{tabular}

Fig. 2 PAUSE protocol for DOAC resumption after a surgery/procedure. ${ }^{\dagger}$ First post-procedure dose delayed at least 24 hours post-procedure; ${ }^{\ddagger}$ first post-procedure dose delayed at least 48 hours post-procedure. Solid arrows indicate usual DOAC dose taken on those days; shaded area indicates no DOAC taken on those days; shaded portion of arrow indicates optional resumption of DOAC on day +2 or +3 . DOAC, direct oral anticoagulant; PAUSE, the Perioperative Anticoagulant Use for Surgery Evaluation.

high risk for venous thromboembolism, the PAUSE protocol permits the use of postoperative thromboprophylaxis with low-dose unfractionated or low-molecular-weight heparin. Similarly, a heparin could be used in patients who cannot take medications by mouth, for example in the setting of a post-operative ileus.

\section{Clinical Outcomes and Rationale}

The primary clinical outcomes are arterial thromboembolism, comprising stroke (ischemic or haemorrhagic), systemic embolism or transient ischemic attack and major bleeding; the secondary outcomes comprise all-cause death, acute coronary syndrome, venous thromboembolism, clinically relevant non-major bleeding and minor bleeding. The period of observation begins from the time of DOAC interruption until 30 days post-procedure. All clinical outcomes are defined based on objective diagnostic criteria (see - Appendix A), and are adjudicated by an independent events adjudication committee, which is blinded to the DOAC patients are receiving.

The rationale for the primary outcomes was to identify thromboembolic events related to atrial fibrillation and anticoagulant interruption and to identify bleeding outcomes that would necessitate either prolonged anticoagulant interruption or re-operation. The classification of nonmajor bleeds into clinically relevant non-major or minor reflected the variable severity of non-major bleeds, for example some requiring medical attention or intervention. We acknowledge that distinguishing between clinically relevant non-major and minor bleeds might pose challenges given that some bleeding is expected in a perioperative setting, especially after major surgery. ${ }^{39}$

\section{Laboratory Outcomes and Rationale}

The primary laboratory outcomes vary according to the DOAC assessed. For dabigatran-treated patients, this is the dTT, as it is considered the most reliable and precise coagulation test to measure the anticoagulant effect of dabigatran. ${ }^{14}$ For rivaroxaban- and apixaban-treated patients, it is DOAC-calibrated anti-factor Xa levels. ${ }^{14}$ The cut-point (50 ng/mL) used to define a clinically acceptable low residual anticoagulant effect was decided upon by consensus among investigators, as there is no widely accepted cut-point that defines a safe level of residual anticoagulation to allow a surgery/procedure to proceed. Alternative cut-points, such as assay-specific lower limits of detection of anticoagulant effect, will also be considered.

The pre-procedure blood samples used in PAUSE are processed at participating clinical sites, using standardized methods, and shipped to the Special Coagulation Laboratory at McMaster University Medical Center for centralized and standardized measurement of the PT, aPTT, TT, dTT and anti-factor Xa levels. The blood processing methods, the coagulation tests used and reference values are described in -Appendix B.

\section{Study Hypothesis and Sample Size Determination}

The primary study hypothesis is that the PAUSE protocol is safe for the perioperative management of each DOAC; that is the risk for major bleeding at a patient level is $1 \%$ and the risk for arterial thromboembolism is $0.5 \%$. The sample size calculation is based on an estimated risk of major bleeding at $1 \%$, with a non-inferiority proportion of $2 \%$ (i.e., the largest proportion that is still considered non-inferior to the reference value of $1 \%$ ). The required sample size is 987 patients per DOAC, which provides $80 \%$ power at the $5 \%$ significance level (one sided). The total number of required patients per DOAC was increased by $10 \%$ to 1,097 to anticipate patients with a cancelled surgery/procedure and those lost to followup. We are more confident about estimates of major bleeding than arterial thromboembolism and, consequently, major bleeding is the primary determinant of sample size, but the sample chosen is such that the expected range of arterial thromboembolism risks is also addressed.

\section{Statistical Analysis Plan}

We shall use descriptive statistics to report on baseline patient characteristics as follows: continuous variables will be reported as median (and interquartile range [IQR]) or mean (and standard deviation), and categorical variables will be reported as frequency or proportions (and 95\% CIs). The proportions, and associated $95 \% \mathrm{CIs}$, for major bleeding and 
arterial thromboembolism will be calculated at the patient level for each DOAC. Non-inferiority tests for one proportion will be performed to examine if, at the patient level, the proportion of major bleeding per DOAC is $<2 \%$ and the proportion of arterial thromboembolism per DOAC is $<1.5 \%$. Additional secondary analyses are planned to assess outcomes according to different DOAC doses, comprising dabigatran 150 and $110 \mathrm{mg}$, rivaroxaban 20 and $15 \mathrm{mg}$, and apixaban 5 and $2.5 \mathrm{mg}$.

For the laboratory outcomes, we will determine in an exploratory manner the median (IQR) of non-specific coagulation test values (PT, aPTT, TT) for all DOACs. We will determine median (IQR) dTT values for patients taking dabigatran and median (IQR) anti-factor Xa levels for patients taking rivaroxaban or apixaban. We will also determine the proportion of patients with dTT and anti-factor Xa levels below certain cut-points, including $<50 \mathrm{ng} / \mathrm{mL}$.

\section{Discussion}

The PAUSE trial is a prospective cohort study that aims to determine if a standardized but patient-specific perioperative management protocol is safe for patients with atrial fibrillation on DOACs who need anticoagulant interruption for an elective surgery/procedure. Safety is defined, for each DOAC, by the management protocol being associated with perioperative risks of major bleeding of $1.0 \%$ ( $80 \%$ power to exclude $2 \%$ ) and risks of arterial thromboembolism of $0.5 \%$ ( $80 \%$ power to exclude $1.5 \%$ ). These risk estimates are similar to those observed in studies involving DOAC- and warfarintreated patients who required perioperative anticoagulant interruption and did not receive heparin bridging. 7,32,40,41

From a study design standpoint, although randomized trials remain the methodological reference standard to investigate pharmacologic or other management interventions, the lack of a plausible comparator group in the domain of perioperative DOAC management precluded adopting such a study design. The prospective cohort design used in PAUSE is also appropriate when expected risks of clinical events are low. Moreover, demonstrating a small treatment effect with different perioperative management strategies when risks of clinical events are expected to be low is unlikely to be clinically meaningful, for example with a $33 \%$ reduction in either major bleeding (from 1.5 to $1.0 \%$ ) or arterial thromboembolism (from 1.0 to $0.67 \%$ ).

The PAUSE study should be considered within the context of related studies assessing the perioperative management of DOAC-treated patients. As shown in - Table 1, there are four sub-studies from the randomized trials that assessed DOACs for stroke prevention in atrial fibrillation in which there was an analysis of perioperative outcomes in patients who required an elective surgery/procedure during the course of these trials. ${ }^{5,40,41}$ However, these were retrospective analyses that did not include standardized perioperative management protocols, with the exception of the RELY study in which a pre-procedure interruption protocol was introduced about half-way through the trial. The prospective Dresden Registry assessed 30-day post-procedure outcomes in 595 rivaroxaban-treated patients who had 863 surgery/ procedures. ${ }^{42} \mathrm{~A}$ limitation of this study was that perioperative DOAC management was not standardized and only $10 \%$ of patients underwent a major procedure. Finally, the prospective dabigatran cohort study is the only study that incorporates a standardized pre- and post-procedure management protocol. ${ }^{11}$ However, this study was limited to patients on dabigatran and only 96 (of 541) patients had a major surgery, in whom more robust data on perioperative management is needed. Finally, none of these studies routinely measured a residual anticoagulant effect at the time of the surgery/procedure. Another study assessed a residual anticoagulant effect after the pre-procedure interruption of dabigatran or rivaroxaban in 65 patients, but the interruption interval, which was not standardized, varied from 1 to 168 hours. $^{43}$

There are two ongoing multi-centre patient registries of DOAC-treated patients who are having an elective surgery/ procedure: the Observatory of Invasive Procedures and Bleeding in Patients Treated with New Oral Anticoagulants (GIHP-NACO, NCT02185027) study in France and the Periprocedural Direct Oral Anticoagulant Management (RAACOD, NCT03182218) study in Spain. Taken together, these patient registries will provide helpful data regarding perioperative outcomes in DOAC-treated patients, but additional prospective cohort studies with standardized anticoagulant management such as PAUSE are needed. To our knowledge, the only other prospective perioperative DOAC management studies (not registered in ClinicalTrials.gov) involve patients with atrial fibrillation who receive only apixaban and patients with prior venous thromboembolism who receive any DOAC. Finally, there are ongoing studies assessing DOAC continuation in patients who are having cardiac pacemaker or implantable cardiac defibrillator procedures, which is an emerging management option with such procedures, but involves patients who differ from those eligible for PAUSE.

There are potential limitations to the PAUSE study design. First, it may not be possible to enrol the same targeted number of patients $(1,092)$ who are receiving dabigatran, rivaroxaban or apixaban. In clinical practice, the global use of each DOAC will vary over time depending on factors that include drug costs, new clinical trial data and drug marketing. The strategy adopted in PAUSE is to continue the study until the targeted sample size is attained for each DOAC so that the safety of the PAUSE protocol can be ascertained for each DOAC with the same statistical power. Second, it may be argued that the interruption interval and decision to proceed to a surgery/procedure should be driven by the residual anticoagulant effect, as measured by DOAC-specific coagulation tests. ${ }^{46}$ In PAUSE, the interruption interval is driven by the pharmacokinetic properties of each DOAC. This approach has been used for the perioperative management of patients who are receiving warfarin or other vitamin $\mathrm{K}$ antagonists, as well as unfractionated or low-molecular-weight heparins, in which laboratory tests at the time of a surgery/procedure are not mandated to ensure minimal or no residual anticoagulant effect. ${ }^{6,13}$ Moreover, requiring laboratory testing prior to a surgery/procedure is problematic because DOAC-specific 


\begin{tabular}{|c|c|c|c|c|c|c|c|c|c|c|c|c|c|c|}
\hline & 峛 & 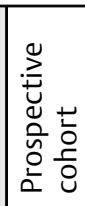 & 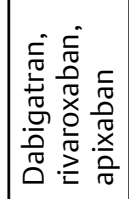 & $\begin{array}{l}\stackrel{8}{ } \\
m \\
m \\
m\end{array}$ & $\left|\begin{array}{l}\stackrel{8}{0} \\
m \\
m\end{array}\right|$ & $\stackrel{\circ}{m}$ & $\stackrel{\rho}{\circ}$ & 吊 & $\stackrel{\check{g}}{\supset}$ & zo & $\stackrel{\check{\nu}}{\nu}$ & $\stackrel{0}{\ominus}$ & $\stackrel{\circ}{\bullet}$ & 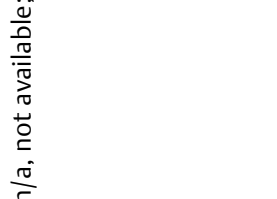 \\
\hline & 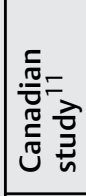 & 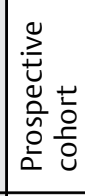 & 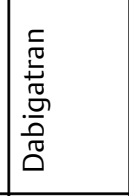 & 文 & $\begin{array}{c}\bar{F} \\
\dot{n}\end{array}$ & ஓे & $\underset{N}{N}$ & $\frac{\pi}{c}$ & $\stackrel{\check{y}}{\rightleftharpoons}$ & z & 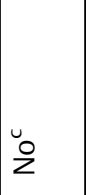 & 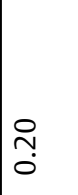 & $\stackrel{\infty}{-}$ & 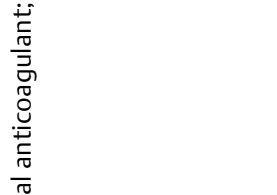 \\
\hline & 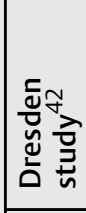 & 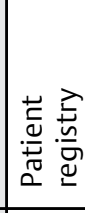 & 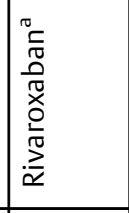 & 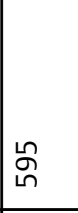 & $\mid \begin{array}{l}\hat{D} \\
\infty\end{array}$ & ஃ̊ํ & $\begin{array}{l}\stackrel{\circ}{+} \\
\stackrel{+}{*}\end{array}$ & $\frac{\pi}{c}$ & z & $\stackrel{\check{\Perp}}{\rightleftharpoons}$ & 2) & 㒸 & $\stackrel{\sim}{\sim}$ & 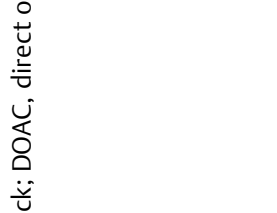 \\
\hline & 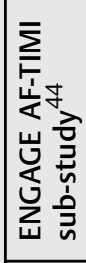 & 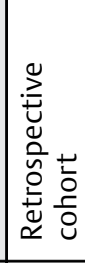 & 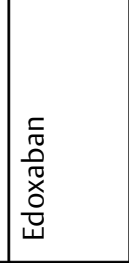 & $\begin{array}{l}\stackrel{\sim}{\alpha} \\
\infty \\
\leftarrow \\
\forall\end{array}$ & $\begin{array}{c}\stackrel{\sim}{0} \\
\infty \\
\leftarrow \\
\forall\end{array}$ & $\stackrel{\curvearrowright}{\text { }}$ & $\frac{\pi}{r}$ & $\stackrel{\infty}{i}$ & z & $\stackrel{\check{\Perp}}{\rightleftharpoons}$ & 운 & $\begin{array}{l}0 \\
0 \\
0\end{array}$ & $\stackrel{\leftrightarrow}{-}$ & 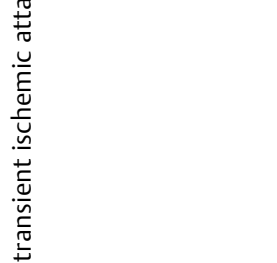 \\
\hline & 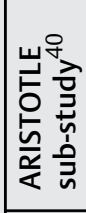 & 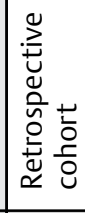 & 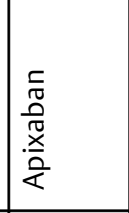 & $\begin{array}{l}8 \\
\varnothing \\
\sigma \\
-\end{array}$ & $\left|\begin{array}{c}\hat{1} \\
\infty \\
i \\
i\end{array}\right|$ & ○ㅇ & $\frac{0}{\Gamma}$ & $\bar{i}$ & z & $\stackrel{\check{\Perp}}{\rightleftharpoons}$ & 운 & $\stackrel{\substack{n \\
m \\
0}}{0}$ & $\mid \begin{array}{l}0 \\
-\end{array}$ & 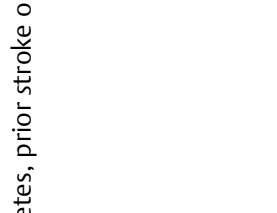 \\
\hline $\bar{\Xi}$ & 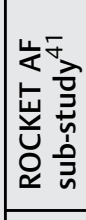 & 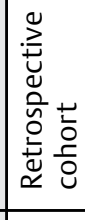 & 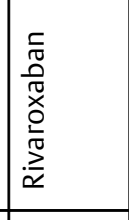 & $\begin{array}{l}n \\
\underline{\omega} \\
i\end{array}$ & $\left|\begin{array}{l}m \\
\tilde{m} \\
\tilde{m}\end{array}\right|$ & $\stackrel{\circ}{\circ}$ & $\begin{array}{l}\stackrel{0}{r} \\
\stackrel{n}{n}\end{array}$ & $\stackrel{\nabla}{\dot{m}}$ & z & $\stackrel{\ddot{\Perp}}{\succ}$ & 운 & 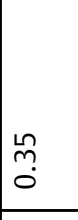 & $\stackrel{\circ}{-}$ & 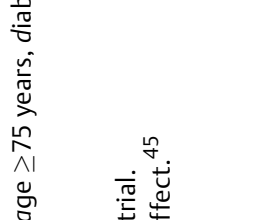 \\
\hline 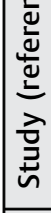 & 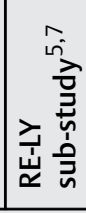 & 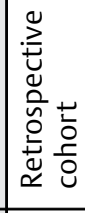 & 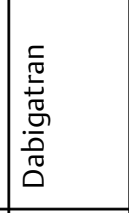 & $\begin{array}{l}5 \\
0 \\
i \\
\end{array}$ & $\begin{array}{l}\overline{5} \\
0 \\
-1 \\
\end{array}$ & 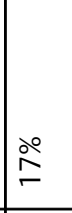 & $\stackrel{\hat{N}}{\stackrel{N}{N}}$ & $\bar{i}$ & $\begin{array}{l}\circ \\
z \\
z\end{array}$ & $\stackrel{\check{\tilde{y}}}{\rightleftharpoons}$ & in & în. & $\begin{array}{l}0 \\
\stackrel{i}{i}\end{array}$ & 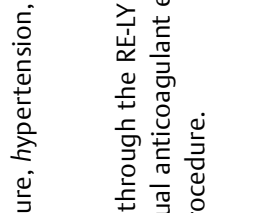 \\
\hline 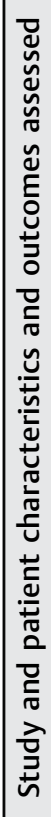 & & 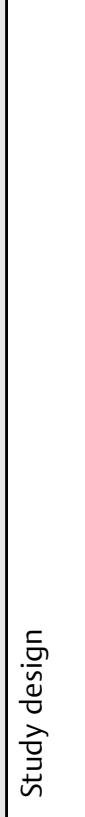 & 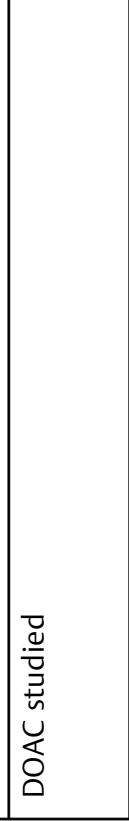 & 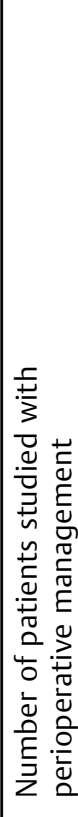 & 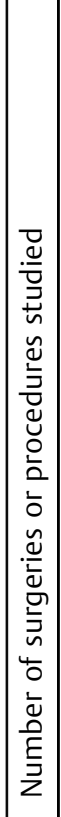 & 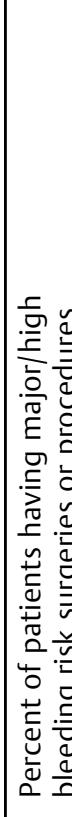 & $\begin{array}{l}> \\
0 \\
0 \\
0 \\
\frac{0}{0} \\
\frac{0}{2}\end{array}$ & 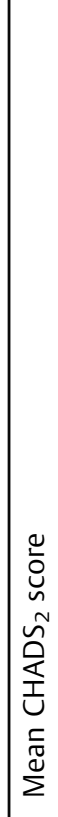 & 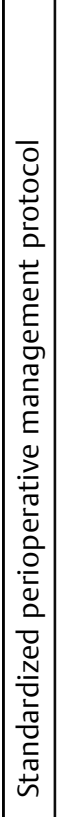 & 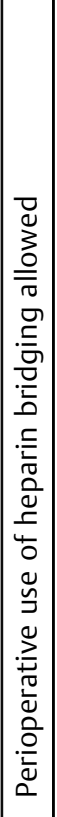 & 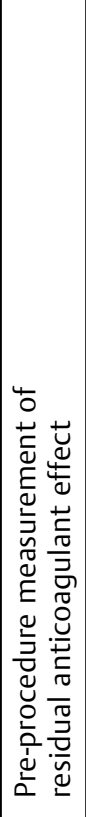 & 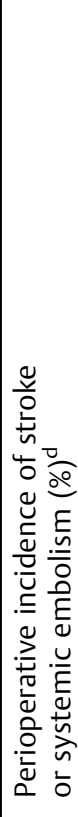 & 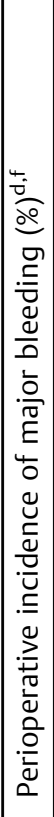 & 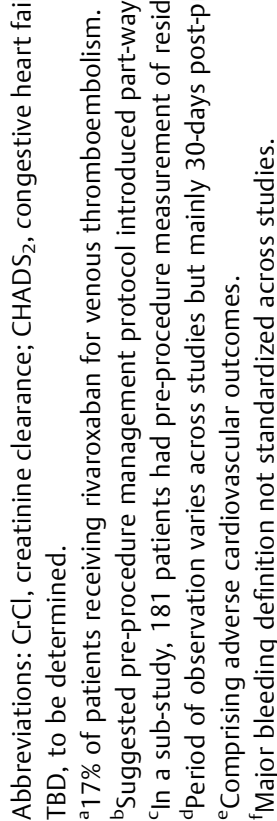 \\
\hline
\end{tabular}


assays are costly, add complexity to pre-surgical assessments, are not routinely available and, even if available, there is uncertainty regarding which assay should be used and what would be considered a safe anticoagulant level cutpoint (e.g. $<50$ or $<30 \mathrm{ng} / \mathrm{mL}$ ). ${ }^{47}$ Nevertheless, this may be an acceptance problem for interventional specialties such as surgery and anaesthesia that routinely perform coagulation testing prior to procedures. Third, there may be underrepresentation of patients at high risk for thromboembolism or those having high bleeding risk surgery or neuraxial anaesthesia. We believe this is unlikely because PAUSE involves a simple, patient-friendly protocol, and we anticipate that a high proportion of eligible patients will be recruited, with adequate representation of various patient and surgery risk categories.

In summary, the PAUSE study will address a common and important unmet clinical need and has the potential to establish a standard-of-care approach for the perioperative management of DOAC-treated patients. The PAUSE management protocol was designed to be easily applied in everyday practice as it is standardized, allowing application across institutions, and also flexible to the real-world eventualities that typify the perioperative clinical setting.

\section{Funding}

The PAUSE study is funded by grants from the Canadian Institutes of Health Research (grant no. 313156) and the Heart and Stroke Foundation of Canada (grant no. G-140006163), and by in-kind support from Aniara-Hyphen Biomed, which provided the DOAC-specific coagulation assays for residual anticoagulation measurements.

\section{Acknowledgments}

We thank the members of the Data Safety Monitoring Board (Drs. Walter Ageno, David Garcia [Chair] and Lehana Thabane) and the members of the Event Adjudication Committee (Drs. Wendy Lim, Lori Linkins, William Ristevski and Demetrios J. Sahlas) for their invaluable contribution to the PAUSE study. We also thank the site study coordinators and research assistants from the McMaster Centre for Transfusion Research, and members of the McMaster University Clinical Research Laboratory and Biobank and Special Coagulation Laboratory at McMaster University Medical Center, for their expert work.

\section{References}

1 Spyropoulos AC, Al-Badri A, Sherwood MW, Douketis JD. Periprocedural management of patients receiving a vitamin K antagonist or a direct oral anticoagulant requiring an elective procedure or surgery. J Thromb Haemost 2016;14(05):875-885

2 Heidbuchel H, Verhamme P, Alings M, et al; Advisors. Updated European Heart Rhythm Association practical guide on the use of non-vitamin-K antagonist anticoagulants in patients with nonvalvular atrial fibrillation: executive summary. Eur Heart J 2016: ehw058

3 Macle L, Cairns J, Leblanc K, et al; CCS Atrial Fibrillation Guidelines Committee. 2016 Focused Update of the Canadian Cardiovascular Society Guidelines for the Management of Atrial Fibrillation. Can J Cardiol 2016;32(10):1170-1185
4 Douketis JD, Spyropoulos AC, Kaatz S, et al; BRIDGE Investigators. Perioperative Bridging Anticoagulation in Patients with Atrial Fibrillation. N Engl J Med 2015;373(09):823-833

5 Healey JS, Eikelboom J, Douketis J, et al; RE-LY Investigators. Periprocedural bleeding and thromboembolic events with dabigatran compared with warfarin: results from the Randomized Evaluation of Long-Term Anticoagulation Therapy (RE-LY) randomized trial. Circulation 2012;126(03):343-348

6 Douketis JD, Berger PB, Dunn AS, et al. The perioperative management of antithrombotic therapy: American College of Chest Physicians Evidence-Based Clinical Practice Guidelines (8th Edition). Chest 2008;133(6, Suppl):299S-339S

7 Douketis JD, Healey JS, Brueckmann M, et al. Perioperative bridging anticoagulation during dabigatran or warfarin interruption among patients who had an elective surgery or procedure. Substudy of the RE-LY trial. Thromb Haemost 2015;113(03):625-632

8 Narouze S, Benzon HT, Provenzano DA, et al. Interventional spine and pain procedures in patients on antiplatelet and anticoagulant medications: guidelines from the American Society of Regional Anesthesia and Pain Medicine, the European Society of Regional Anaesthesia and Pain Therapy, the American Academy of Pain Medicine, the International Neuromodulation Society, the North American Neuromodulation Society, and the World Institute of Pain. Reg Anesth Pain Med 2015;40(03):182-212

9 Doherty JU, Gluckman TJ, Hucker WJ, et al. 2017 ACC Expert Consensus Decision Pathway for Periprocedural Management of Anticoagulation in Patients With Nonvalvular Atrial Fibrillation: a report of the American College of Cardiology Clinical Expert Consensus Document Task Force. J Am Coll Cardiol 2017;69(07): 871-898

10 Raval AN, Cigarroa JE, Chung MK, et al; American Heart Association Clinical Pharmacology Subcommittee of the Acute Cardiac Care and General Cardiology Committee of the Council on Clinical Cardiology; Council on Cardiovascular Disease in the Young; and Council on Quality of Care and Outcomes Research. Management of patients on non-vitamin K antagonist oral anticoagulants in the acute care and periprocedural setting: a scientific statement from the American Heart Association. Circulation 2017;135(10): e604-e633

11 Schulman S, Carrier M, Lee AY, et al; Periop Dabigatran Study Group. Perioperative management of dabigatran: a prospective cohort study. Circulation 2015;132(03):167-173

12 Siegal D, Yudin J, Kaatz S, Douketis JD, Lim W, Spyropoulos AC. Periprocedural heparin bridging in patients receiving vitamin $\mathrm{K}$ antagonists: systematic review and meta-analysis of bleeding and thromboembolic rates. Circulation 2012;126(13):1630-1639

13 Douketis JD, Spyropoulos AC, Spencer FA, et al. Perioperative management of antithrombotic therapy: antithrombotic therapy and prevention of thrombosis, 9th ed: American College of Chest Physicians Evidence-Based Clinical Practice Guidelines. Chest 2012;141(2, Suppl):e326S-e350S

14 Samuelson BT, Cuker A, Siegal DM, Crowther M, Garcia DA. Laboratory assessment of the anticoagulant activity of direct oral anticoagulants: a systematic review. Chest 2017;151(01): 127-138

15 Van Blerk M, Bailleul E, Chatelain B, et al. Influence of dabigatran and rivaroxaban on routine coagulation assays. A nationwide Belgian survey. Thromb Haemost 2015;113(01):154-164

16 Tripodi A, Padovan L, Veena C, Scalambrino E, Testa S, Peyvandi F. How the direct oral anticoagulant apixaban affects thrombin generation parameters. Thromb Res 2015;135(06):1186-1190

17 Vandenbroucke JP, von Elm E, Altman DG, et al; STROBE Initiative. Strengthening the Reporting of Observational Studies in Epidemiology (STROBE): explanation and elaboration. PLoS Med 2007; 4(10):e297

18 Euser AM, Zoccali C, Jager KJ, Dekker FW. Cohort studies: prospective versus retrospective. Nephron Clin Pract 2009;113(03): c214-c217 
19 Cockcroft DW, Gault MH. Prediction of creatinine clearance from serum creatinine. Nephron 1976;16(01):31-41

20 Connolly SJ, Ezekowitz MD, Yusuf S, et al; RE-LY Steering Committee and Investigators. Dabigatran versus warfarin in patients with atrial fibrillation. N Engl J Med 2009;361(12):1139-1151

21 Granger CB, Alexander JH, McMurray JJ, et al; ARISTOTLE Committees and Investigators. Apixaban versus warfarin in patients with atrial fibrillation. N Engl J Med 2011;365(11):981-992

22 Patel MR, Mahaffey KW, Garg J, et al; ROCKET AF Investigators. Rivaroxaban versus warfarin in nonvalvular atrial fibrillation. N Engl J Med 2011;365(10):883-891

23 Giugliano RP, Ruff CT, Braunwald E, et al; ENGAGE AF-TIMI 48 Investigators. Edoxaban versus warfarin in patients with atrial fibrillation. N Engl J Med 2013;369(22):2093-2104

24 Gladstone DJ, Geerts WH, Douketis J, Ivers N, Healey JS, Leblanc K. How to monitor patients receiving direct oral anticoagulants for stroke prevention in atrial fibrillation: a practice tool endorsed by Thrombosis Canada, the Canadian Stroke Consortium, the Canadian Cardiovascular Pharmacists Network, and the Canadian Cardiovascular Society. Ann Intern Med 2015;163(05):382-385

25 Spyropoulos AC, Douketis JD. How I treat anticoagulated patients undergoing an elective procedure or surgery. Blood 2012;120 (15):2954-2962

26 Clark NP, Witt DM, Davies LE, et al. Bleeding, recurrent venous thromboembolism, and mortality risks during warfarin interruption for invasive procedures. JAMA Intern Med 2015;175(07): 1163-1168

27 Skeith L, Taylor J, Lazo-Langner A, Kovacs MJ. Conservative perioperative anticoagulation management in patients with chronic venous thromboembolic disease: a cohort study.J Thromb Haemost 2012;10(11):2298-2304

28 Baron TH, Kamath PS, McBane RD. New anticoagulant and antiplatelet agents: a primer for the gastroenterologist. Clin Gastroenterol Hepatol 2014;12(02):187-195

29 Witt DM, Delate T, McCool KH, et al; WARPED Consortium. Incidence and predictors of bleeding or thrombosis after polypectomy in patients receiving and not receiving anticoagulation therapy. J Thromb Haemost 2009;7(12):1982-1989

30 Gage BF, Waterman AD, Shannon W, Boechler M, Rich MW, Radford MJ. Validation of clinical classification schemes for predicting stroke: results from the National Registry of Atrial Fibrillation. JAMA 2001;285(22):2864-2870

31 Lip GY, Nieuwlaat R, Pisters R, Lane DA, Crijns HJ. Refining clinical risk stratification for predicting stroke and thromboembolism in atrial fibrillation using a novel risk factor-based approach: the euro heart survey on atrial fibrillation. Chest 2010;137(02): 263-272

32 Steinberg BA, Peterson ED, Kim S, et al; Outcomes Registry for Better Informed Treatment of Atrial Fibrillation Investigators and Patients. Use and outcomes associated with bridging during anticoagulation interruptions in patients with atrial fibrillation: findings from the Outcomes Registry for Better Informed Treatment of Atrial Fibrillation (ORBIT-AF). Circulation 2015;131(05): 488-494

33 Stangier J, Rathgen K, Stähle H, Gansser D, Roth W. The pharmacokinetics, pharmacodynamics and tolerability of dabigatran etexilate, a new oral direct thrombin inhibitor, in healthy male subjects. Br J Clin Pharmacol 2007;64(03):292-303
34 Stangier J, Rathgen K, Stähle H, Mazur D. Influence of renal impairment on the pharmacokinetics and pharmacodynamics of oral dabigatran etexilate: an open-label, parallel-group, single-centre study. Clin Pharmacokinet 2010;49(04):259-268

35 Kubitza D, Becka M, Wensing G, Voith B, Zuehlsdorf M. Safety, pharmacodynamics, and pharmacokinetics of BAY 59-7939-an oral, direct Factor Xa inhibitor-after multiple dosing in healthy male subjects. Eur J Clin Pharmacol 2005;61(12):873-880

36 Cui Y, Song Y, Wang J, et al. Single- and multiple-dose pharmacokinetics, pharmacodynamics, and safety of apixaban in healthy Chinese subjects. Clin Pharmacol 2013;5:177-184

37 Douketis JD, Syed S, Schulman S. Periprocedural management of direct oral anticoagulants: comment on the 2015 American Society of Regional Anesthesia and Pain Medicine Guidelines. Reg Anesth Pain Med 2016;41(02):127-129

38 Dunn AS, Spyropoulos AC, Turpie AG. Bridging therapy in patients on long-term oral anticoagulants who require surgery: the Prospective Peri-operative Enoxaparin Cohort Trial (PROSPECT). J Thromb Haemost 2007;5(11):2211-2218

39 Schulman S, Angerås U, Bergqvist D, Eriksson B, Lassen MR, Fisher W; Subcommittee on Control of Anticoagulation of the Scientific and Standardization Committee of the International Society on Thrombosis and Haemostasis. Definition of major bleeding in clinical investigations of antihemostatic medicinal products in surgical patients. J Thromb Haemost 2010;8(01):202-204

40 Garcia D, Alexander JH, Wallentin L, et al. Management and clinical outcomes in patients treated with apixaban vs warfarin undergoing procedures. Blood 2014;124(25):3692-3698

41 Sherwood MW, Douketis JD, Patel MR, et al; ROCKET AF Investigators. Outcomes of temporary interruption of rivaroxaban compared with warfarin in patients with nonvalvular atrial fibrillation: results from the rivaroxaban once daily, oral, direct factor Xa inhibition compared with vitamin $\mathrm{K}$ antagonism for prevention of stroke and embolism trial in atrial fibrillation (ROCKET AF). Circulation 2014;129(18):1850-1859

42 Beyer-Westendorf J, Gelbricht V, Förster K, et al. Peri-interventional management of novel oral anticoagulants in daily care: results from the prospective Dresden NOAC registry. Eur Heart J 2014;35(28):1888-1896

43 Godier A, Martin AC, Leblanc I, et al. Peri-procedural management of dabigatran and rivaroxaban: Duration of anticoagulant discontinuation and drug concentrations. Thromb Res 2015;136 (04):763-768

44 Douketis J, Weitz J, Murphy SA, et al. Perioperative adverse outcomes in patients with atrial fibrillation taking edoxaban or warfarin: Analysis of the ENGAGE AF-TIMI 48 Trial. J Am Coll Cardiol 2015;65(10S):A2092

45 Douketis JD, Wang G, Chan N, et al. Effect of standardized perioperative dabigatran interruption on the residual anticoagulation effect at the time of surgery or procedure. J Thromb Haemost 2016;14(01):89-97

46 Tripodi A. To measure or not to measure direct oral anticoagulants before surgery or invasive procedures. J Thromb Haemost 2016; 14(07):1325-1327

47 Spyropoulos AC, Al-Badri A, Sherwood MW, Douketis JD. To measure or not to measure direct oral anticoagulants before surgery or invasive procedures: comment. J Thromb Haemost 2016;14(12):2556-2559 


\section{Appendix A: Clinical Outcomes and Diagnostic Criteria}

\section{Primary Clinical Outcomes}

The first primary outcome is major bleeding, defined by $\geq 1$ of the following criteria: (1) bleeding that is fatal or is symptomatic and retroperitoneal, intracranial, intraspinal, intraocular, pericardial, intramuscular with compartment syndrome or intra-articular; (2) non-surgical bleeding causing a drop in haemoglobin to $\geq 20 \mathrm{~g} / \mathrm{L}$ (1.24 mmol/L) or leading to transfusion of $\geq 2$ units whole blood or red cells within 24 hours of bleeding; (3) surgical bleeding that leads to intervention (e.g. re-operation) or has one of the following: interferes with mobilization, leads to delayed wound healing or leads to deep wound infection; (4) surgical site bleeding that is unexpected and prolonged and/or sufficiently large to cause hemodynamic instability associated with a drop in haemoglobin $\geq 20 \mathrm{~g} / \mathrm{L}(1.24 \mathrm{mmol} / \mathrm{L})$ or transfusion of $\geq 2$ units whole blood or red cells within 24 hours of bleeding. The second primary outcome is arterial thromboembolism, comprising (1) ischemic stroke, defined as any new focal neurologic deficit that persists for $>24$ hours or any new focal neurologic deficit of any duration that occurs with evidence of acute infarction on computed tomography (CT) or magnetic resonance imaging (MRI) of the brain; (2) systemic embolism, defined as symptomatic embolism to upper or lower extremity or abdominal organ, confirmed intraoperatively or by objective imaging studies (e.g. CT angiography) and (3) transient ischemic attack, defined as symptomatic focal neurologic deficit (lasting typically $<1$ hour) that occurs with no evidence of acute infarction on CT/MRI of the brain.

\section{Secondary Clinical Outcomes}

The secondary clinical outcomes comprise (1) clinically relevant non-major bleeding, defined as bleeding not satisfying the criteria for major bleeding that requires a medical assessment (e.g. unscheduled visit to the doctor's office or to an emergency department) and/or treatment/intervention; (2) minor bleeding, defined as bleeding not satisfying the criteria for major or clinically relevant non-major bleeding; (3) death due to any cause; (4) venous thromboembolism, defined by symptomatic deep vein thrombosis or pulmonary embolism that is confirmed by objective imaging studies (e.g. ultrasound, CT pulmonary angiogram) and (5) acute coronary syndrome, defined by symptomatic myocardial ischemia that is confirmed by objective criteria (electrocardiographic and/or elevated cardiac troponins).

\section{Appendix B: Blood Processing METHODS and Coagulation Assays Used}

The pre-procedure blood sample is collected into a Vacutainer tube (Becton Dickinson Canada, Mississauga, Ontario, Canada) containing sodium citrate $(0.105 \mathrm{M}, 3.2 \%)$; it is centrifuged for 15 minutes at $1,500 \mathrm{~g}$, plasma is transferred and double-spun at $1,500 \mathrm{~g}$ for 5 minutes to ensure platelet poor plasma $\left(<10 \times 10^{9} / \mathrm{L}\right.$ platelets). Platelet-poor plasma is separated into aliquots and stored at $-70^{\circ} \mathrm{C}$ at each participating clinical site. Plasma samples are shipped to and are analysed at the Hamilton Regional Laboratory Medicine Program's Special Coagulation Laboratory by medical laboratory technologists who are blinded to patient characteristics.

The following coagulation function tests and assays are being used: prothrombin time (PT; Siemens Thromborel S, Marburg, Delaware, United States); activated partial thromboplastin time (aPTT; Siemens Dade Actin FS, Marburg, Delaware, United States); thrombin time (TT; Sigma-Aldrich, Oakville, Canada); dilute thrombin time (dTT) (Hemoclot, Hyphen BioMed, Neuville-sur-Oise, France) and anti-factor Xa levels (Hyphen BioMed, Neuville-sur-Oise). All testing was performed on a STArEvolution analyser (Diagnostica Stago, Asnières-sur-Seine, France). The reference intervals for these assays are as follows: PT $=11-15$ seconds; INR $=0.8-1.2 ;$ aPTT $=22-35$ seconds; $\mathrm{TT}=20-30$ seconds. There is no therapeutic range for dTT and anti-factor Xa levels, but the lower limit that is reported is $<20 \mathrm{ng} / \mathrm{mL}$ for the dTT and for the rivaroxaban and apixaban antifactor Xa levels. 